\title{
IN VITRO AND IN VIVO ASSESSMENT OF ENAMEL COLOUR STABILITY IN TEETH TREATED WITH LOW-VISCOSITY RESIN INFILTRATION - A LITERATURE REVIEW
}

\author{
Karolina Polak-Kowalska, Elżbieta Pels \\ Chair and Department of Paediatric Dentistry, Medical University in Lublin, Poland
}

\begin{abstract}
Infiltration with a low-viscosity resin is one of the methods of minimally invasive treatment of enamel-induced hypo-mineralisation. It is one of the most modern methods of treatment of white lesions, which can be a bridge between remineralisation therapy and surgical methods. Long-term aesthetic effects of treatment depend on the colour stability of the resin used in this method. It is believed that due to the physical properties of the resin used in the infiltration method, there may be changes in the colour of the surface of the enamel subjected to infiltration. The paper attempts to summarise current reports regarding the colour stability assessment of enamel treated by infiltration with a low-viscosity resin confirmed in in vitro and in vivo studies. The previous results of laboratory tests indicate that the resin used in the infiltration method is susceptible to discolouration of internal and external origin. On the other hand, clinical trials prove that maintaining stable aesthetic effects of infiltration treatment is possible. It is assumed that the extent of discolouration may vary depending on the patient's eating and hygiene habits. Therefore, prophylaxis has a strong impact on the quality of infiltrated surfaces. The aim of the work is to summarise current knowledge on the stability of the colour of the enamel surface treated by resin infiltration, based on a review of the selected literature. The analysis of the literature is also aimed at presenting the methods proposed by clinicians in the event of a change in the colour of the teeth subjected to the infiltration procedure.
\end{abstract}

KEY WORDS: discoloration, colour stability, colour changes, infiltrated surfaces, resin infiltration.

J Stoma 2019; 72, 3: 135-141

DOI: https://doi.org/10.5114/jos.2019.87534

\section{INTRODUCTION}

Resin infiltration is an emerging technique for management of non-cavitated lesions [34]. It is a bridge between preventive and invasive treatment, postponing as long as possible the need for reconstruction and the subsequent need to replace fillings [15].

The concept of this method is based on infiltration with a light-cured resin of demineralised tissues, aimed at creating a diffusion barrier for acids and dissolved minerals inside the damaged structure and ensuring its strengthening and mechanical stabilisation [33].
The resin fills the micropores in the damaged enamel and can therefore lead to slowing or stopping the progress of caries [24].

Resin infiltration is classified as a minimally invasive method of treatment because it requires removal of only the superficial enamel layer with $15 \%$ hydrochloric acid to a depth of $30-58 \mathrm{~nm}[32,42]$. The highly mineralised outer layer of the enamel could hinder the penetration of the resin into the interior of the change, so it is necessary to eliminate it [42].

The resin used in this method has very low density and viscosity, low contact angle, and high surface ten-
JOURNAL OF STOMATOLOGY CZASOPISMO STOMATOLOGICZNE
AdDRess FOR CORRESPONDENCE: dr hab. n. med. Elżbieta Pels, Chair and Departament of Pediatric Dentistry, Medical University in Lublin, 7 Karmelicka St., 20-081 Lublin, Poland, e-mail: elzbieta.pels@umlub.pl

ReCEIVED: 26.04.2019 • ACCEPTED: 24.07.2019 • PUblished: 08.08.2019 
sion [36]. These properties are desirable characteristics that allow the resin to penetrate the enamel micropores due to capillary forces [6].

The infiltration method is used to treat carious lesions without the loss of enamel occurring on the smooth surfaces of the teeth and proximal ones reaching up to the outermost third of the dentin (radiologically to the level of D1) [25, 31]. Recently, the range of indications has been extended to include the use of infiltration to mask pre-eruptial changes, such as mild and moderate fluorosis, traumatic hypo-mineralisation, and molar incisor hypo-mineralisation (MIH) $[4,7,10,11,28]$. According to many authors, using the infiltration method is an effective treatment for all kinds of white lesions within the enamel, regardless of their aetiology [4].

Minimally invasive treatment with resin infiltration is an alternative to previously used methods of treatment of hypo-mineral enamel changes, especially those located in the aesthetic zone, and is more and more often recommended for clinical use [11].

In aesthetic dentistry most of all, its additional property is used, which is the ability to improve the aesthetic effect of white enamel changes using optical methods. The principle of masking white changes with a resin is based on a change in light scattering [7]. This is due to the fact that an infiltrant exhibiting a refractive index (1.52) similar to a healthy enamel (1.62), improves the transmission of photons through insufficiently mineralised enamel and restores its translucency [32]. The difference in the refractive index between infiltrated and healthy enamel is insignificant; therefore, the treated lesions regain transparency and become similar to the surrounding enamel $[26,32]$.

ICON (DMG, Germany), used for infiltration, was used for the first time in 2009 [18].

In the literature there are numerous reports on the effectiveness of the method of resin infiltration in inhibiting the progress of caries and masking of white spots $[12,17,19,32,35]$. Some physical and mechanical properties of infiltration such as the assessment of infiltration depth, micro-hardness, and resistance to acids have also been examined [21, 42]. However, as far as colour stability and resistance to discolouration of infiltrated enamel are concerned, few papers have been published so far, and this subject is still under investigation [1].

\section{OBJECTIVE}

The aim of this work is to review current knowledge about the stability of the colour of the enamel surface treated by resin infiltration based on the selected literature. The aim of the analysis is to assess the colour durability of tooth enamel treated by infiltration with a low-viscosity resin (ICON DMG, Hamburg, Germany) confirmed in in vitro and in vivo tests and possible treatment methods in case of discolourations. The causes of colour change of infiltrated surfaces and risk factors affecting the occurrence of discolorations were also discussed.

\section{MATERIAL AND METHODS}

The PubMed, Medline, and Embase databases were used to search for literature on the colour stability of enamel treated by infiltration. The following keywords were used: resin infiltration, icon resin infiltration, staining agents, white spot lesions, color stability, surface roughness, color change, white defects on enamel, proximal caries lesions, enamel carious lesions. The analysis included original articles, randomised clinical trials, case-control studies, and literature reviews published until 2019. The criterion of inclusion in the review was the compliance of the information with the specific work objectives evaluated.

\section{RESULTS}

A total of 1320 articles were obtained, mainly in English. Publications not directly related to the subject of research were excluded. Finally, 43 items covering issues related to the review were qualified for the review. The article discusses the results of eight in vitro and four in vivo studies on the colour stability of infiltrated surfaces. The work includes articles published in the years 2006-2019.

\section{STABILITY OF COLOUR OF INFILTRATED SURFACES AND DISCOLOURATION - AETIOLOGY}

Because the treatment of white enamel changes often affects the aesthetic zone, colour stability, i.e. preserving its colour in the oral environment by the resin, is an important determinant of the long-term success of the treatment. Possible colour change and susceptibility to discolouration can be a big problem and lead to dissatisfaction of the patient [8].

The aetiology of tooth colour change after infiltration treatment can be multifactorial. These can be both internal and external factors $[1,8]$.

One of the internal factors is the type of resin that is part of the preparation used in the infiltration method. The resin used for infiltration mainly consists of tetra-ethylene glycol dimethacrylate (TEGDMA) with high penetration efficiency $[6,33,36]$. TEGDMA resin is often used in aesthetic dentistry. However, it is known that, compared to other resins such as UDMA and Bis-GMA, it has the highest degree of water and other fluid absorption due to the presence of hydrophilic ether bonds and the presence of unreacted methacrylate in the resin matrix $[1,6,33,36,43]$. Other resin ingredients, such as ter- 
tiary amines or aliphatic amines that accelerate the polymerisation under the influence of light or heat, may undergo an effect of shadowing [8]. In addition, variable temperatures in the mouth can cause degradation of resin-hydroxyapatite bonds due to the difference in thermal expansion coefficients between hydroxyapatite and the resin [43]. It can affect the integrity of infiltrated surfaces and contribute to the formation of microcracks and micro-joints [43]. Another factor that may contribute to increased susceptibility to discolouration is polymerisation shrinkage [22]. According to Lee et al., studies have shown that a lower filler content in the resin results in a greater polymerisation shrinkage and thus contributes to increased micro-leakage, and so the resin may have a greater tendency to absorb colouring substances [22]. Hence, the hydrophilicity and degradation of the polymer matrix in the oral environment as well as the polymerisation shrinkage may contribute to deterioration of the physical and mechanical properties of the resin [8]. As a consequence, it may lead to internal discolouration and problems with the colour stability of the treated surfaces [43].

Discolouration of infiltrated surfaces may also be caused by external factors [6]; they are considered to be the main cause of discolouration of resin-based materials $[2,6,11,43]$. Outer discolouration is often observed in patients who smoke and consume products rich in tannins $[1,8]$.

It is believed that the increased susceptibility to external discolouration of infiltrated surfaces with nicotine and food and beverage colourants may be caused by the degradation of the superficial resin layer and an increase in roughness [41]. Furthermore, Ulrich et al., indicate that the rough surface may cause increased colonisation of the biofilm on the surface, infiltrated enamel, and eventually cause further demineralisation of the enamel and biodegradation of the resin structure [40].

According to the authors, the rough surface of the enamel can be a place of increased risk of bacterial adhesion and plaque accumulation compared to smoother surfaces and consequently may lead to demineralisation and development of secondary caries $[2,16,40]$.

Studies have shown that the threshold surface roughness value above which accumulation occurs in plaque is $200 \mathrm{~nm}(0.2 \mathrm{~lm})[2,16]$.

According to Paris et al., and in accordance with the manufacturer's recommendations, excess polymer should be removed before polymerisation [29]. Otherwise, a thin layer of resin may contribute to the accumulation of plaque, including the accumulation of external dyes [29]. However, in a study by Gurdogan et al. it was observed that despite the removal of excess material and polishing, the infiltrated surfaces were characterised by a rough structure [16]. The values were more than $0.2 \mathrm{~lm}$ and were significantly higher than in the control group, which was calmed down by healthy enamel [16, 40]. Also, a study carried out by Ulrich et al. showed greater roughness of infiltrated surfaces compared to a healthy glaze, despite the removal of excess material before polymerisation and polishing treatment [40].

The first studies conducted by Taher et al. assessing the degree of roughness of infiltrated surfaces showed no statistically significant difference between infiltrated and healthy enamel [37]. However, subsequent studies using other test methods showed a non-homogeneous surface compared to a healthy control enamel [38].

These changes in surface integrity resulting both from the physical properties of the resin and the interaction of the infiltrate with the biofilm lead to the formation of porosity, cracks, and surface roughness [40]. This vicious circle caused further colonisation of bacteria, and thus an increase in the risk of absorption of exogenous dyes contained in the diet is possible [40].

\section{ANALYSIS OF LITERATURE}

It should be highlighted that although the white spot masking effects are observed immediately, some doubt remains with regard to the stability of aesthetic outcomes resulting from the exposure to staining agents and the ageing of the low-viscosity resin used for infiltration. Undoubtedly, long-term colour stability, i.e. the ability of the resin to retain its colour in an oral environment, remains questionable [22]. This issue is an interesting research problem; however, so far only a few publications on this subject have been created [1].

Evaluation of the tooth's colour can be carried out, among others, using instrumental techniques such as colourimetry, spectrophotometry, image analysis, or through visual assessment [6]. Because spectrophotometry allows an objective assessment of tooth colour, this method is the most commonly used in the presented studies $[1,6,19,26]$.

Numerous hypotheses suggest that enamel subjected to the infiltration procedure is susceptible to the influence of colouring agents contained in the diet. Thus, the colour stability of these surfaces may be impaired. The prevailing view in the literature is that some beverages such as tea, coffee, and wine as well as tobacco can cause discolouration [2].

Studies carried out by Paris et al. on the enamel of bovine teeth show that polishing the infiltrated enamel causes resistance to colourants. The authors explain this by reducing the surface porosity and removing the oxygen inhibition layer [30]. Not all authors confirm this regularity.

Cohen-Carneiro et al. published the results of an in vitro study on the enamel of bovine teeth [8]. The durability of the enamel colour treated with resin infiltration was evaluated. The demineralised enamel and enamel subjected to remineralisation was also evaluated for colour and brightness after the exposure. All samples immersed in coffee changed in colour and brightness over time, includ- 
ing polished, infiltrated enamel. Among the experimental groups, the remineralisation group showed the least colour change after exposure to coffee, statistically different from all other groups. It also seems interesting that this study showed a greater change in colour in other groups (demineralised enamel and demineralised enamel subjected to infiltration), but without statistically significant differences between each other [8].

Also, from the results of a study conducted by Araujo et al. on the enamel of bovine teeth, coffee staining resulted in a significant change in the colour of both demineralised and infiltrated treated enamel. Similarly as in the previous study, it turned out that the examined groups did not show any differences from each other after staining [1].

Borges et al., in an in vitro study carried out on artificially, induced demineralisation changes in the enamel of bovine teeth and showed that the exposure of healthy enamel, demineralised enamel, enamel subjected to remineralisation, and enamel subjected to infiltration in coloured solutions resulted in significant changes in colour [6]. However, in contrast to the results of CohenCorneiro et al. and Araujo et al., demineralised enamel treated with resin and polishing showed a significantly greater colour change compared to other groups [6].

An evaluation of the colour durability of infiltrated surfaces on human teeth in vitro was carried out by Leland et al. The authors found a significant change in the colour of the resin after exposure to colourants. Regardless of the type of colouring agent from the spectrophotometer, it was found that the polished, infiltrated areas showed greater sensitivity to staining as compared to demineralised, non-infiltrated enamel [23].

Zhao et al., in an in vitro study also on human teeth, similarly to previous authors, proved that infiltrated enamel showed a greater change in colour compared to healthy enamel [43]. The study showed a much lower tendency of infiltrated enamel for discolouration compared to demineralised enamel, which remains in contradiction with the results obtained by Leland et al. [43]. Interesting information is also provided by the results of the study by Lee et al., carried out on human teeth, but with the use of other research methods [22]. Under a light microscope the penetration depth of external dyes in heat-treated and demineralised enamel not subjected to infiltration was assessed. In demineralised enamel, dyes penetrated deeper than enamel subjected to infiltration, and this was a statistically significant difference [22]. Therefore, in accordance with the assumptions of the infiltration technique, the resin blocks or limits diffusion paths for dissolved minerals and microorganisms, but also for potential colourants.

The above in vitro studies were carried out on both bovine teeth and human teeth. Research conducted by Attitia et al. showed that the chemical and physical properties of bovine enamel, such as composition, density, and micro-hardness, are similar to those of human enamel $[1,5,6]$. It was also found that bovine enamel has a similar potential to absorb colouring substances from food as human enamel [6]. However, the results of the tests showed some discrepancies in the sensitivity of the enamel to the colouring agents.

As the authors themselves noticed, contradictory results of research may result from certain limitations of the research carried out. Although in most of the presented studies a spectrophotometer was used to assess the colour of the teeth, in the tests substances with different staining potential and different concentrations were used. The studies also differed in time of staining and length of observation. Therefore, it should be assumed that the degree of sensitivity of infiltrated enamel to colourants may depend on the intensity of dyeing and the time of exposure to colourants.

Some authors, including Gurdogan et al., suggest that it may be necessary to modify the procedure for polishing infiltrated enamel [16]. A smooth surface could prevent possible complications in the form of superficial discolouration. The results of the conducted studies clearly show that in order to ensure the long-term success of resin treatment, further clinical trials are necessary to improve infiltrated surfaces $[16,26]$. Otherwise, due to the properties of the resin used in the infiltration method, the accumulation of plaque and food dyes on the surface enamel may be unavoidable [16].

While the vast majority of authors agree that enamel subjected to infiltration may change colour after exposure to colourants in vitro due to the type of resin used and the rough surface that is characterised by the enamel subjected to infiltration, the test results in vivo do not seem to confirm this hypothesis.

The results of in vivo studies to date indicate that maintaining stable aesthetic effects of infiltration is possible. This is indicated by the results of some clinical trials.

The longest observation so far was conducted by Knösel et al. on a group of 20 patients with demineralisation changes of enamel after permanent treatment with an orthodontic appliance [20]. Spectrophotometer testing showed colour stability of the treated surfaces for up to 24-45 months. Although testing with a spectrophotometer showed a slight change in the colour and brightness of the treated surfaces during individual follow-up visits compared to baseline values, these differences were not statistically significant. In addition, according to the authors, these values were so low that they were not visible to the naked eye in clinical conditions. The camouflage effect did not change over the entire observation period [20].

Eckstein et al., also conducted a comprehensive study evaluating the effects of treating white spots by means of resin infiltration [13]. Twenty people with enamel demineralisation changes resulting from permanent orthodontic appliance treatment participated in the study. Using a spectrophotometer, the colour and brightness of the treated surfaces were evaluated six and 12 months 
after the procedure. Based on the research, they found that the infiltration material and the obtained effects of white spot treatment remained stable for 12 months in vivo. The authors did not find a statistically or clinically significant change in colour and brightness throughout the observation period, which allows for the correct estimation of the durability of aesthetic effects [13].

An in vivo study carried out by Feng et al., assessing inter alia the colour of infiltrated surfaces, showed stability of the aesthetic effect after a 12-month observation period. The study involved eight patients after permanent treatment with an orthodontic appliance, in which enamel demineralisation was diagnosed. Teeth images were analysed using image analysis software (Image-pro plus 6.0) [14].

19-month clinical observations conducted by Tirlet et al. on a group of four patients treated by infiltration with resin due to enamel developmental changes also showed aesthetic appearance of the treated surfaces throughout the observation period [39].

It is also noteworthy that in none of the above studies did patients report adverse events or side effects during follow-up visits.

Borges et al. and Cohen-Carneiro et al. noted that the results of the in vitro study cannot be directly compared to clinical conditions $[6,8]$. Borges et al. noted that in clinical conditions saliva buffer capacity and mechanical cleaning of the tooth surface can cause a greater resistance of the resin to colouring agents [6]. Similarly, the likelihood of external discolouration and its extent may vary depending on the composition of the saliva and the patient's habits, such as oral hygiene, diet, and stimulants (smoking) $[1,6,22]$.

Unfortunately, in none of the presented studies, despite the use of objective assessment methods, did the authors take into account the impact of diet and individual habits in the studied patients, such as smoking and consumption of colouring products, on the obtained results. Consumption of colouring substances could be different for individual patients. In the long-term forecast of colour stabilisation of infiltrated surfaces, hygienic and nutritional habits, including consumption of colouring substances in the studied group, should be carefully considered $[1,13,20]$.

It is interesting to note that in the studies conducted by Cohen-Carneiro et al., Paris et al., Borges et al., and Leland et al. it was shown that polishing of infiltrated surfaces reduced the effects of dyeing $[6,8,23,30]$. The polishing treatment caused restoration of brightness in all studied groups; however, as shown by the study conducted by Cohen-Corneiro et al., the best effect was obtained on surfaces subjected to remineralisation [8].

Araujo et al. also report that in the case of external discolouration of the infiltrated surface, it is possible to remove these changes by means of whitening [1]. In an in vitro study of bovine teeth, it was found that bleaching demineralised enamel treated with $16 \%$ urea peroxide gel resulted in the removal of a significant part of discolouration [1]. After bleaching demineralised enamel showed the highest brightness value compared with other groups, but no significant difference between the control group and the infiltrated group. The whitening procedure caused a significant improvement in the appearance of both healthy enamel and enamel subjected to infiltration [1].

The results of an in vitro study carried out by Villalta et al. also confirm that it is possible to completely remove stains from composite resins using bleaching [41].

Therefore, it can be assumed that if the discolouration of the infiltrate occurs, the polishing or bleaching treatment can be successfully applied. Both of these treatments have a strong effect on reducing discolouration.

It is worth noting that external dyes also build into the structure of demineralised enamel and, as a consequence, can also lead to unsightly brown discolouration on their surface $[6,30]$.

Research conducted by Yazkan et al., showed that the roughness value of infiltrated enamel was actually higher than that of healthy enamel, but significantly lower compared to demineralised enamel [42].

The dentist must, therefore, decide whether it is more beneficial for the patient to immediately infiltrate the diagnosed lesions or rather leave them to spontaneous remineralisation. This decision should take into account oral hygiene, as well as the patient's fears and expectations regarding the appearance of teeth [20]. Patients with lower expectations regarding aesthetics and bad hygiene may benefit from remineralising therapy described in detail in the literature and confirmed in in vivo studies $[3,9,27]$. Cohen-Corneiro et al. reported that the remineralised enamel proved to be the least sensitive to colouring substances [8]. Further clinical studies are necessary to assess the impact of roughness on the degree of bacterial plaque accumulation and the possibility of external discolouration. The small number of long-term clinical observations carried out to date does not allow this theory to be excluded.

Long-term clinical observations are difficult due to problems with patients getting check-ups within the prescribed dates. However, long-term in vivo observation of infiltrated surfaces is necessary to estimate the durability of potential aesthetic effects. The review of the literature on this subject shows that patients awaiting aesthetic effects but with good oral hygiene should be qualified for the resin infiltration procedure.

According to a study conducted by Leland et al., Araujo et al., and Borges et al., wine and coffee showed the highest potential for dyeing $[1,23]$. In turn, the research conducted by Lee et al. shows that the greater tendency to stain was from ice tea and grape juice [22]. In addition, the authors drew attention to the fact that products with a low $\mathrm{pH}$ may adversely affect the integrity of infiltrated surfaces [22]. This theory is also confirmed by the results of the study carried out by Ritwik 
et al. [34]. Therefore, it is worth emphasising that, according to current knowledge, patients should, if possible, avoid or minimise the intake of infiltration acidic and colouring food products to increase the colour fastness of the resin used in this method $[21,22,43]$.

\section{CONCLUSIONS}

The aim of this work was to present the results of in vitro and in vivo tests regarding the assessment of tooth colour durability after infiltration with a low-viscosity resin. An attempt was made to find the cause of colour disorders of the enamel treated by the method of infiltration and the best procedures in the case of discolouration.

The results of the above in vitro tests indicate that the surface of the enamel subjected to infiltration may be discoloured under the influence of colouring agents. In clinical settings the extent of discolouration may vary depending on the patient's habits, such as oral hygiene and diet. Patients should avoid consuming colouring products to minimise the risk of external discolouration. Prevention has an influence on the quality of infiltrated surfaces. In the case when there are changes in the colour of the infiltrated surface, it is possible to remove these changes even with the help of polishing or bleaching.

In order to ensure the long-term success of the resin treatment, further in vivo tests are necessary to more accurately assess surface integrity and roughness in relation to the colour stability of the resin used in the infiltration method and the risk of external and internal discolouration. The results of the review of the literature indicate the need to modify, above all, the procedure for polishing infiltrated enamel because its rough surface was considered the main cause of the discolouration.

\section{CONFLICT OF INTEREST}

The authors declare no potential conflicts of interest with respect to the research, authorship, and/or publication of this article.

\section{References}

1. Araújo GSA, Naufel FS, Alonso RCB, Lima DANL, Puppin-Rontani RM. Influence of staining solution and bleacching on color stability of resin used for caries infiltration. Oper Dent 2015; 40: E250-256.

2. Arnold WH, Meyer AK, Naumova EA. Surface roughness of initial enamel caries lesions in human teeth after resin infiltration. Open Dent J 2016; 10: 505-515.

3. Asokan S, Geethapriya PR, Vijayasankari V. Effect of nonfluoridated remineralizing agents on initial enamel carious lesions: a systematic review. Indian J Dent Res 2019; 30: 282-290.

4. Attal JP, Atlan A, Denis M, Vennat E, Tirlet G. White defects on enamel: diagnosis and anatomopathology: two essential factors for proper treatment (part 2). Int Orthod 2014; 12: 1-31.

5. Attia ML, Aguiar FH, Mathias P, Ambrosano GM, Fontes CM, Liporoni PC. The effect of coffee solution on tooth color during home bleaching applications. Am J Dent 2009; 22: 175-179.
6. Borges A, Caneppele T, Luz M, Pucci C, Torres C. Color stability of resin used for caries infiltration after exposure to different staining solutions. Oper Dent 2014; 39: 433-440.

7. Borges AB, Caneppele TMF, Masterson D, Maia LC. Is resin infiltration an effective esthetic treatment for enamel development defects and white spot lesions? A systematic review. J Dent 2017; 56: 11-18.

8. Cohen-Carneiro F, Pascareli AM, Christino MR, Vale HF, Pontes DG. Color stability of carious incipient lesions located in enamel and treated with resin infiltration or remineralization. Int J Paediatr Dent 2014; 24: 277-285.

9. Creeth JE, Karwal R, Hara AT, Zero DT. A randomized in situ clinical study of fluoride dentifrices on enamel remineralization and resistance to demineralization: effects of zinc. Caries Res 2018; 52: 129-138.

10. Crombie F, Manton D, Palamara J, Reynolds E. Resin infiltration of developmentally hypomineralised enamel. Int J Paediatr Dent 2014; 24: 51-55.

11. Denis M, Atlan A, Vennat E, Tirlet G. White defects on enamel: diagnosis and anatomopathology: two essential factors for proper treatment (part 1). Int Orthod 2013; 11: 139-165.

12. Doméjean S, Ducamp R, Léger S, Holmgren C. Resin infiltration of non-cavitated caries lesions: a systematic review. Med Princ Pract 2015; 24: 216-221.

13. Eckstein A, Helms HJ, Knösel M. Camouflage effects following resin infiltration of postorthodontic white-spot lesions in vivo: one-year follow-up. Angle Orthod 2015; 85: 374-380.

14. Feng CH, Chu XY. Efficacy of one year treatment of icon infiltrant resin on post-orthodontic white spots. Beijing Da Xue Xue Bao 2013; 45: 40-43.

15. Gelani R, Zandona AF, Lippert F, Kamocka MM, Eckert G. In vitro progression of artificial white spot lesions sealed with an infiltrant resin. Oper Dent 2014; 39: 481-488.

16. Gurdogan EB, Ozdemir-Ozenen D, Sandalli N. Evaluation of surface roughness characteristics using atomic force microscopy and inspection of microhardness following resin infilration. J Esthet Restor Dent 2017; 29: 201-208.

17. Hallgren K, Akyalcin S, English J, Tufekci E, Paravina RD. Color properties of demineralized enamel surfaces treated with a resin infiltration system. J Esthet Restor Dent 2016; 28: 339-346.

18. Kielbassa AM, Muller J, Gernhardt CR. Closing the gap between oral hygiene and minimally invasive dentistry: a review on the resin infiltration technique of incipient (proximal) enamel lesions. Quintessence Int 2009; 40: 663-681.

19. Kim S, Kim EY, Jeong TS, Kim JW. The evaluation of resin infiltration for masking labial enamel white spot lesions. Int J Paediatr Dent 2011; 21: 241-248.

20. Knösel M, Eckstein A, Helms HJ. Long-term follow-up of camouflage effects following resin infiltration of post orthodontic whitespot lesions in vivo. Angle Orthod 2019; 89: 33-39.

21. Kumar H, Palamara JEA, Burrow MF, Manton DJ. An investigation into the effect of a resin infiltrant on the micromechanical properties of hypomineralised enamel. Int J Paediatr Dent 2017; 27: 399-411.

22. Lee J, Chen JW, Omar S, Kwan SR, Meharry M. Evaluation of stain penetration by beverages in demineralized enamel treated with resin infiltration. Oper Dent 2016; 41: 93-102.

23. Leland A, Akyalcin S, English JD, Tufekci E, Paravina R. Evaluation of staining and color changes of resin infiltration system. Angle Orthod 2016; 86: 900-904.

24. Mandava J, Reddy YS, Kantheti S, Chalasani U, Ravi RC, Borugadda R, Konagala RK. Microhardness and penetration of artificial white spot lesions treated with resin or colloidal silica infiltration. J Clin Diagn Res 2017; 11: ZC142-ZC146.

25. Martignon S, Ekstrand KR, Gomez J, Lara JS, Cortes A. Infiltrating/sealing proximal caries lesions: a 3-year randomized clinical trial. J Dent Res 2012; 91: 288-292.

26. Mazur M, Westland S, Guerra F, Corridore D, Vichi M, Maruotti A, Nardi GM, Ottolenghi L. Objective and subjective aesthetic performance of Icon treatment for enamel hypomineralization 
lesions in young adolescents: a retrospective single center study. J Dent 2018; 68: 104-108.

27. Mendes AC, Restrepo M, Bussaneli D, Zuanon AC. Use of casein amorphous calcium phosphate (CPP-ACP) on white-spot lesions: randomised clinical trial. Oral Health Prev Dent 2018; 16: 27-31.

28. Muñoz MA, Arana-Gordillo LA, Gomes GM, Gomes OM, Bombarda $\mathrm{NH}$, Reis A, Loguercio AD. Alternative esthetic management of fluorosis and hypoplasia stains: blending effect obtained with resin infiltration techniques. J Esthet Restor Dent 2013; 25: 32-39.

29. Paris S, Meyer-Lueckel H, Mueller J, Hummel M, Kielbassa AM. Progression of sealed initial bovine enamel lesions under demineralizing conditions in vitro. Caries Res 2006; 40: 124-129.

30. Paris S, Schwendicke F, Keltsch J, Dorfer C, Meyer-Lueckel H. Masking of white spot lesions by resin infiltration in vitro. J Dent 2013; 41 Suppl 5: e28-e34.

31. Paris S, Soviero VM, Seddig S, Meyer-Lueckel H. Penetration depths of an infiltrant into proximal caries lesions in primary molars after different application times application times in vitro. Int J Paediatr Dent 2012; 22: 349-355.

32. Prasada KL, Penta PK, Ramya KM. Spectrophotometric evaluation of white spot lesion treatment using novel resin infiltration material (ICON). J Conserv Dent 2018; 21: 531-535.

33. Rahiotis C, Zinelis S, Eliades G, Eliades T. Setting characteristics of a resin infiltration system for incipient caries treatment. J Dent 2015; 43: 715-719.

34. Ritwik P, Jones CM, Fan Y, Sarkar NK. Hydrolytic and color stability of resin infiltration: a preliminary in vitro trial. J Contemp Dent Pract 2016; 7: 377-381.

35. Rocha Gomes Torres C, Borges AB, Torres LM, Gomes IS, de Oliveira RS. Effect of caries infiltration technique and fluoride therapy on the colour masking of white spot lesions. J Dent 2011; 39: 202-207.

36. Subramaniam P, Girish Babu KL, Lakhotia D. Evaluation of penetration depth of a commercially available resin infiltrate into artificially created enamel lesions. An in vitro study. Conserv Dent 2014; 17: 146-149.

37. Taher NM, Alkhamis HA, Dowaidi SM. The influence of resin infiltration system on enamel microhardness and surface roughness: an in vitro study. Saudi Dent J 2012; 24: 79-84.

38. Taher NM. Atomic force microscopy and tridimensional topography analysis of human enamel after resinous infiltration and storage in water. Saudi Med J 2013; 34: 408-414.

39. Tirlet G, Chabouis HF, Attal JP. Infiltration, a new therapy for masking enamel white spots: a 19-month follow-up case series. Eur J Esthet Dent 2013; 8: 180-190.

40. Ulrich I, Mueller J, Wolgin M, Frank W, Kielbassa AM. Tridimensional surface roughness analysis after resin infiltration of (deproteinized) natural subsurface carious lesions. Clin Oral Investig 2015; 19: 1473-1483.

41. Villalta P, Lu H, Okte Z, Garcia-Godoy F, Powers JM. Effects of staining and bleaching on color change of dental composite resins. J Prosthet Dent 2006; 95: 137-142.

42. Yazkan B, Ermis RB. Effect of resin infiltration and microabrasion on the microhardness, surface roughness and morphology of incipient carious lesions. Acta Odontol Scand 2018; 76: 473-481.

43. Zhao X, Ren YF. Surface properties and color stability of resin-infiltrated enamel lesions. Oper Dent 2016; 41: 617-626. 